Syed, M., \& Kathawalla, U. K. (2022). Cultural psychology, diversity, and representation in open science. In K. C. McLean (Ed.), Cultural methods in psychology: Describing and transforming cultures (pp. 427-454). New York: Oxford University Press.

\title{
Cultural Psychology, Diversity, and Representation in Open Science
}

\author{
Moin Syed \& Ummul-Kiram Kathawalla \\ University of Minnesota
}

\begin{abstract}
The current moment in psychology is one of great challenges and great opportunities. The open science movement--the move towards more transparent, credible, and reproducible science--has led to a redefinition of what constitutes "normal science." However, the field of cultural psychology, broadly construed, has by and large not engaged with the open science movement, and likewise, the open science movement has by and large not engaged with cultural psychology. The purpose of the present chapter is to bring open science and cultural psychology closer together, highlighting how they can benefit one another. In doing so, we focus our discussion on three types of representations regarding diversity in psychological research and how they intersect with open science: representation of researchers, or the diversity of the scientists actually doing the research, representation of samples, or who is included as participants in our research studies, and representation of perspectives, or the substantive conceptual and theoretical views we bring to our work. For each of these three types of representation we outline the problem, and then discuss how embracing the principles and behaviors of open science can help.
\end{abstract}

Keywords: Cultural psychology, open science, meta-science, diversity, ethnic minority psychology

Movements come and go, some fizzle and become distant memories, whereas others have lasting power that effect actual change, creating a new normal. We are inarguably embroiled in a major movement in psychology - the open science movement-which involves themes and issues that have been raised throughout the history of the field (Gelman, 2016; Syed, 2019). And yet signs point to things being different this time; that the open science movement is not just the latest trend or fad in the field but that the current zeitgeist represents a period of massive instability as we redefine

We thank Neil Lewis Jr. and Kate McLean for thoughtful and helpful comments on an earlier draft of this chapter. Some of the ideas expressed in this chapter were presented as a talk titled, "Stop Talking About 'Diversity' in Open Science" at the 2020 Society for the Improvement of Psychology Science preconference. That talk, and other information associated with this work can be found at https://osf.io/ndzea/. This document was typeset using Wiernik's (2019) \#prettypreprint template.

Moin Syed \& Ummul-Kiram Kathawalla, Department of Psychology, University of Minnesota.

Correspondence concerning this paper should be addressed to Moin Syed, Department of Psychology, University of Minnesota, 75 East River Road, Minneapolis, MN 55455. Email: moin $a$,umn.edu "normal science" (Kuhn, 1962; see Nelson et al., 2018; Nosek et al., 2018; Spellman, 2015).

Taking this premise to be true-that the open science movement is reshaping normal science-means that the movement is relevant to the work of all scientists, and thus all scientists should be closely attending to how open science should impact their work. In reality, however, the awareness, acceptance, and implementation of open science is uneven across the sciences broadly and across subfields within psychology. This unevenness in aligning with the open science movement may result in an asymmetry of rigor, where some areas of the field have more rigorous standards and expectations that come through engaging in open science practices compared to others who follow bygone practices.

It is our contention that the field of cultural psychology, broadly construed, has by and large not engaged with the open science movement. Importantly, we likewise contend that the open science movement has by and large not engaged with cultural psychology. This latter issue is a point of departure between our current effort and most other published works on open science in different domains of psychology. The most common 
framing is why and how different fields would benefit from adopting open science practices (e.g., McBee et al., 2018 for giftedness research; Sakaluk, 2016 for sexual science; Syed, 2020b for identity research). Indeed, there is an excellent article on replication and reproducibility in cross-cultural psychology (Milfont \& Klein, 2018), but it is entirely focused on applying open science to cross-cultural psychology. Much less common are treatments of how open science would benefit from engaging with specific fields.

The purpose of the present chapter is to bring open science and cultural psychology closer together, highlighting how they can benefit one another. One of the major barriers to making progress on aligning cultural psychology and open science is that both are broad terms that subsume many varieties of perspectives and behaviors, and so any productive discussion about these issues must include clear definitions. A key point with regard to these terms is that all of them are so broad that they are generally unhelpful when attempting to make any specific points, and that more narrow terms and concepts are most appropriate in any given application. Thus, a major goal of this chapter is to bring greater specificity to the discussion. Moreover, in this chapter we adopt a perspective that places issues of culture and diversity at the center of the discussion. That is, rather than seeing culture and diversity as extra or optional, we argue that such issues are foundational and can infuse all aspects of research, especially within an open science framework.

To this end, the first section of this chapter includes definitions and explanations of cultural psychology and open science, respectively. In the second section we draw upon cultural psychology to focus our discussion on three types of representations and how they intersect with open science: representation of researchers, or the diversity of the scientists actually doing the research, representation of samples, or who is included as participants in our research studies, and representation of perspectives, or the substantive conceptual and theoretical views we bring to our work. For each of these three types of representation we outline the problem, and then discuss how embracing the principles and behaviors of open science and cultural psychology can mutually benefit each other. In this way, we seek to both identify many of the looming issues at the intersection of cultural psychology and open science as well as provide concrete suggestions for how to better align the two in practice.

\section{Cultural Psychology, Open Science, and Their Intersections}

\section{The Varieties of Cultural Psychology}

As we have previously articulated (Syed \& Kathawalla, 2018), cultural psychology is best understood as a broad family of approaches to psychological research. We will only briefly summarize these different perspectives here, and the interested reader is directed to the more detailed discussion in Syed and Kathawalla (2018). The term cultural psychology can pertain to at least six different perspectives. The first three themselves use the term cultural psychology to refer to a) sociocultural research focused on how people construct meaning within specific activities and contexts (e.g., Rogoff, 2003; see also Manago, Santer, Barsigian, \& Walsh, this volume), b) discursive approaches focused on hyper-contextual cognitive processes situated within social power structures (e.g., Muller Mirza \& Dos Santos Mamed, 2019), and c) social psychological approaches that use comparative designs to examine divergent psychological processes across cultural contexts (e.g., Markus \& Kitayama, 1991). Cultural psychology can also be used to describe indigenous psychology, which shares the meaning-focused perspective of cultural psychology but draws more heavily on localized knowledge and practices and tends to have minimal interest in generalizing beyond that context (Shweder, 2000). In contrast to cultural and indigenous psychology, cross-cultural psychology was founded within a universalist model, aiming to identity invariance and commonality in psychological processes (typically those identified in Western contexts) through cross-cultural comparisons (e.g., Segall et al., 1998). A final type of cultural psychology is ethnic minority psychology, which is specifically focused on the meaning and experience of being an ethnic minority within a specific national context (e.g., Hall et al., 2011). Ethnic minority psychology tends to have an explicit emphasis on power, oppression, and privilege.

Thus, cultural psychology itself is a vague term that can correspond to many different theoretical perspectives and methodological approaches. One common theme among them is that they place an emphasis on diversity. In some ways this is not helpful, though, as diversity itself is a vague term that can pertain to just about any form of human variation (e.g., race, ethnicity, gender, immigrant status, geographic, ideological). 
Indeed, cultural psychology, like many areas, has its own challenges that it continually tries to address, beyond just the lack of specificity. Cultural psychology is not always as inclusive as it could be, especially when considering international representation and minority representation outside of North America. Some researchers may have limited access to diverse samples based on race, ethnicity, SES, and/or ability, which therefore limits their ability to study certain research questions--or the access they do have is restricted, leading to underpowered studies. Finally, in cultural psychology there can be limited perspectives that frame our thinking, perspectives that do not always benefit robust knowledge generation (see discussion in Syed and Kathawalla (2018) on research on collectivist and individualistic countries). These three challenges map on to the three representations that are the focus of this chapter - researchers, samples, and perspectives - and which we argue open science can help with.

\section{The Open Science Movement}

Open science is a broad term that encompasses a host of specific beliefs and behaviors (Spellman et al., 2018; Yarkoni, 2019). There is no agreed-upon definition of open science, and because of the variety of ideas that fall under the broad label, the lack of specificity means that having discussions about "diversity in open science" can be challenging.

In an attempt to synthesize existing definitions and concepts, Syed (2019) defined open science as a set of principles and behaviors that promote transparent, credible, reproducible, and accessible science. This definition puts forward four principles--transparency, credibility, reproducibility, and accessibility-each of which can be manifest as specific behaviors. This definition, specifically the separation of principles and behaviors, is attractive for highlighting the fact that open science is applicable to all kinds of research, no matter the theories, methods, or topics under study. This approach is meant to eschew a "one size fits all" mentality. The four principles are broadly applicable to any area of study, but the specific behavioral manifestations will always be contextualized by the research question and methodology (Crüwell et al., 2019).

Briefly, transparency pertains to researchers being honest about theoretical, methodological, and analytic decisions made throughout the research cycle; credibility is the degree of trustworthiness and believ- ability of the research reported in the literature; reproducibility pertains to how well we keep records of what we do, at all phases of the research cycle, so that everything can be reproduced when needed (not if needed, because there will always be a need for reproducibility); and accessibility pertains to making all aspects of the research cycle open and available for those who are interested.

The latter principle of accessibility speaks directly to diversity and representation, and yet diversity is not a strong point of discussion within the movement. For example, recent editorials and essays on open science do not make any mention of increasing diversity in the movement (e.g., Lewis, 2019; Renkewitz \& Heene, 2019). As we discuss more below, diversity efforts are sometimes perceived to be stymied by the false notion that there is only one "right" way to do open science, which is inflexible and time consuming and may limit researchers from other regions of the world or disciplines to believe they can be involved in the movement (Allen \& Mehler, 2019; Bahlai et al., 2019; Kathawalla et al., 2020). The discussions that are present tend to be diffuse and wide-ranging, and thus there is a great need to carefully examine the intersections of cultural psychology and open science.

\section{Intersections of Cultural Psychology and Open Science}

Some of the challenges in the field of cultural psychology and the open science movement can be addressed by better fusion of the two areas together. A major question is how to go about doing this. On the face, it may seem like the two could be at times incompatible. For example, the replicability of studies has been a major focus of the open science movement. Some varieties of cultural psychology, especially those in the sociocultural, discursive, and indigenous traditions, tend to be more aligned with a constructivist or critical paradigm, in which replication is often not necessary or even expected. This example highlights the need to be mindful of the breadth of both open science and cultural psychology; open science is not entirely, or even primarily, concerned with replication, and cultural psychology is not all conducted within constructivist or critical paradigms. Nevertheless, most types of cultural psychology place emphasis on meaning, interpretation, and deep description. Understanding, rather than replication, is the primary focus. If such an approach was brought to mainstream psychology, which is largely situated within a postpositivistic paradigm that seeks to uncover truisms 
about the world (see Delucio \& Villicana, this volume), it is possible we would see greater rates of replication because we would have spent more time better understanding the nature of our constructs and the relations among them. In this chapter we take an even broader stance, that just thinking about culture and diversity as central to our collective work can generally benefit open science practice, and vice versa.

Given that we are cultural psychologists involved in open science, it may be surprising that diversity is not one of the core principles of open science we outlined above. This is for two reasons. First, as noted, there has been a surprising lack of formal writing on the intersections of diversity, cultural psychology, and open science. This could be, in part, because the open science movement in psychology began within social psychology. Despite the fact that social psychology tends to study topics such as prejudice and discrimination, the field does so primarily using White samples and using mainstream experimental methods (Hartmann et al., 2013; Hunt et al., 2000; Roberts et al. 2020; Syed et al., 2018). Moreover, although social psychology focuses heavily on contextualized psychological processes, universality of those contextualized processes is treated as the default. As an illustration, in the wake of the many replication failures reported in the Reproducibility Project: Psychology (Open Science Collaboration, 2015), van Bavel et al. (2016) purported to demonstrate that such failures could be explained because of the "contextual sensitivity" of social psychology, that observed effects will vary by time, culture, or location. Putting aside the fact that their analysis did not actually provide evidence for that position (see Inbar, 2016), such claims of contextual sensitivity or "hidden moderators" (Simonsohn, 2017) are only made after a failed replication, whereas the original studies tend to make unconstrained universal claims (see also Simons et al., 2017). A field that generally assumes universality unless demonstrated otherwise is unlikely to take a cultural psychological perspective as its starting point (Delucio \& Villicana, this volume). All in all, a consumer of the open science literature in psychology might conclude that diversity has not been even a peripheral perspective within the movement.

The second, more central reason for diversity not being a separate principle is because we reject what is sometimes referred to as the "diversity as chapter 13" model. This model refers to the fact that edited collections often begin with "basic processes," move into topic domains, and then end with context, diversity, and culture. Rarely are diversity and culture viewed as part of "basic processes." This model converges with the "universal unless demonstrated otherwise" perspective previously described. In contrast, we adopt an infused perspective, or to extend the metaphor, "diversity as chapter 1" (see Fish \& Syed, 2018; Goodnow, 2011; Juang et al., 2012; Rogoff, 2003). Rather than diversity being a separate principle that one can choose whether or not to align with, this perspective takes the position that diversity issues are relevant and inextricably linked with all other principles. This perspective runs counter to the view that diversity and other concerns are part of a zero-sum game. To put it another way, there is no open science without diversity.

For example, a collaborative opinion piece published in American Scientist focusing on diversity and open science included a heavy emphasis on the principle of accessibility (Bahlai, et al., 2019). Notably, none of the authors were psychologists. The authors pushed back on what they perceive as the "all or nothing" attitude of open science proponents, arguing that some researchers can face constraints in their ability to engage in some open science practices. For example, publishing in open access journals can be expensive, and thus researchers who do not have access to publications funds via grants or institutional support do not have the same ability to publish open access. Indeed, their piece was largely focused on issues of access, resource inequalities, and power differentials due to institutional affiliations and career stage. However, they also addressed credibility and reproducibility, arguing that researchers may preregister a study and make all study materials available, but are not able to openly share their data due to ethical or privacy concerns.

In recent years there has been strong push back on the "all or nothing" view of open science, with many researchers arguing for a more selective approach (Whitaker, 2018; Corker, 2018; Kathawalla et al., 2020; Nuijten, 2019; Syed, 2019). This ecumenical approach to open science, when seen as a virtuous position, solves many of the concerns raised by Bahlai et al (2019). It does not, however, directly address the looming question that has heretofore received insufficient attention: how should we think about culture, diversity, and open science in psychology?

As described previously, we view the growing endorsement of these principles and enactments of associated behaviors as constituting a movement. One of the premier analyses of social movements comes from the interpretative framework of intersectionality (see Delucio \& Villicana, this volume). In articulating the 
concept, Crenshaw (1993) highlighted how the U.S. Civil Rights Movement was largely a movement by and for Black men, and the Women's Movement was largely a movement by and for White women. As a result, the unique issues faced by Black women within these movements was rendered invisible. Indeed, intersectional invisibility is a psychological concept that has since been advanced to highlight the psychological significance of not sufficiently attending to power dynamics and who is included and excluded (Cole, 2009; Purdie-Vaughns \& Eibach, 2008; Syed et al., 2018).

This intersectional analysis can be applied to the open science movement by posing the questions of who is included, who is excluded, and what are the implications of such. In this chapter we take up the minority view, exploring what open science is, what it is not, and what it can be for cultural psychology. As will become clear, we believe that cultural psychology should be part of the open science movement. Open science people should attend to cultural psychology, and cultural psychology people should attend to open science. It is a case of mutual accommodation.

Because our focus is on the open science movement and who is included, we organize our discussion around three types of representation: representation of researchers, or the diversity of the scientists actually doing the research, representation of samples, or who is included as participants in our research studies, and representation of perspectives, or the substantive conceptual and theoretical views we bring to our work. These three representations can - and often do-intersect in important ways, but we also see value in considering each one separately. More importantly, we believe it is important to be mindful that these are separate instantiations of representations/diversity, and one is not a substitute for another nor are they in competition with one another. Finally, we have previously discussed many of these issues with respect to ethnic minorities in psychology in general (Syed, 2017; Syed et al., 2018; Syed \& Kathawalla, 2018), thus here our focus is largely circumscribed by the context of the open science movement. When discussing each of the three, we first lay out the problem, and then highlight some ways that the open science movement and cultural psychology can work together to help address the problem.

\section{Representation of Researchers}

Psychology has traditionally been a hierarchical field controlled by a relatively small elite group of researchers and institutions, and is thus best described as an oligarchy (see Hamby, this volume; Wilson, Breen $\&$ DuPré, this volume). This oligarchy has served a major gatekeeping function in the field, restricting access to grants, publications, and jobs. Not surprisingly, ethnic minority researchers have not, by and large, been a part of the oligarchy.

The open science movement is a direct threat to this structure of the field. The principles of transparency and accessibility lead to another desirable feature of science: accountability. The open science movement seeks to lay bare the processes by which achievements come to be, so that we can have confidence that research and scholarly activity was rewarded based on its merits rather than networks. The movement strives to be a democratic approach, where all can participate regardless of career stage, institution, or country of residence, and thus seeks to break down traditional power structures. For this reason, it is not surprising that major critics of open science practices tend to be more senior researchers who have enjoyed success under the traditional model (e.g., Baumeister, 2016; Fiske, 2016; Strack, 2017).

Importantly, the aforementioned description is most accurately described as the promise of the open science movement. The movement, if implemented in accordance with its underlying principles, has the real power to create a more democratic approach to science. But there is good reason to think that it will not. Without intentional action, any movement will likely reproduce any existing power imbalances that it was not designed to break down. The open science movement was not conceived as a social-structural movement, in that issues of racial and gender-based power dynamics were never core to the movement. Accordingly, without explicit attention to these issues, the scientific community will once again "move on" without seriously incorporating them into their work.

Indeed, most of the discussions about diversity and open science have consisted of online chatter or at diversity "hack-a-thons" at Society for the Improvement of Psychology Science (SIPS) meetings. The majority of these online discussions and hack-a-thons have focused on diversity with respect to inclusion, specifically whether the "open science community" is a welcoming place for individuals who identify with marginalized groups (see also Masuzzo, 2020). This is a tricky issue to discuss and summarize here, as we 
are mostly referring to discussions in academic spheres of Twitter and Facebook, and thus tend to be half-formed thoughts and assertions pushed out to the broader academic community. We find value in noting these discussions here, though both because such discussions have shaped the online discourse around open science and diversity and to highlight the limited spheres in which diversity and open science have been discussed. Moreover, as media scholar Sarah Jackson $(2019,2020)$ has highlighted, Twitter has amplified the voices of groups marginalized due to race, gender, sexuality, and so on, providing a platform to be heard and initiate change. Accordingly, Twitter plays an important function in democratizing science, as scholars with less access to power and resources can have their contributions recognized. Indeed, the first SIPS diversity hack-a-thon came about because of a tweet by Neil Lewis, Jr. (2017) requesting such a discussion. All of that said, Academic Twitter and Facebook are very small, non-representative slices of the psychology research community, with those engaging in open science being an even smaller share. Thus, issues pertaining to diversity and open science have scantly been discussed when considering the full field of psychological research.

\section{How Greater Synergy between Open Science and Cultural Psychology Can Contribute to Represen- tation of Researchers}

As noted, the under-representation of researchers from marginalized backgrounds is a structural problem that requires structural solutions. The open science movement is a structural solution, but focused on a different problem. And thus, if we carry on as we are, the social-structural nature of the "previous science" will carry on to the new.

Within open science, the principle of accessibility is intended to directly challenge the social-structural context, and thus that is a site for altering attitudes and practices that can promote the diversity of researchers. Within psychology, accessibility has largely been discussed in terms of making articles available via open access or preprints. Thus, we need to collectively broaden how we think about accessibility. For example Albornoz \& Chan (2018), stated, "We must ask: what assumptions are embedded in narratives about Open Science, whose interests are they serving and in turn whose interests are neglected in this framing?" (p. 75). This analysis of power is fundamental to an intersectional perspective on how science operates (see Syed et al., 2018).
Albornoz and Chan (2018) discuss how the Open Science movement has mainly highlighted EuroAmerican scholarship, and similarly how the main organizations such as Center for Open Science and FOSTER are focused on Euro-American scholarship (see also Chan, 2019; Vicente-Saez \& MartinezFuentes, 2018). This results in voices of other researchers in other regions of the world to be neglected. It is important to think about what assumptions are assumed with the Open Science movement and how the interests may or may not serve "diverse" researchers, samples, or theoretical perspectives.

The Open and Collaborative Science in Development Network (OCSDNet), an international research network, studied the ways in which the Open Science movement has contributed towards equality. Specifically, the broad research question for the network was "whether and under what conditions, open and collaborative science practices could lead to development outcomes and community well-being" (p. 8; Chan et al., 2019). This research project found some examples of how Open Science can create space for political and social change, albeit outside of psychology. For example, research teams in Colombia and Costa Rica worked with coffee-plantation farmers, drawing on the principles of openness to share scientific knowledge with the farmers and to promote social and environmental well-being in the production process (Albornoz \& Chan, 2018). In another example, a team based in Lebanon developed low-cost technologies and methods to measure water contamination, and again the purpose was to spread the knowledge openly (Albornoz \& Chan, 2018). These examples may not be the conventional Open Science principles considered in psychology, but they emphasize the importance of representation of researchers in the movement and changing the way we think about and enact our research.

A major change in the distribution of resources is central to reforming the power structure in the field. As we discuss in the subsequent sections, greater engagement in data sharing and collaboration can lead to increased opportunities for scholarships for researchers with less access to resources. Moreover, we discuss how changing both how we assign credit for publication (creditorship vs. authorship) and how we make decisions about what to publish (moving towards Registered Reports) would help equalize the field. Research funding is of course a major barrier for many under-represented scholars (e.g., Ginther et al., 2012 for Black scholars and NIH funding). A relatively new intervention to address this issue are "grant 
lotteries," in which funders randomly select grant applications for funding that meet a predetermined quality threshold (see Adam, 2019). Doing this ensures that disparities in access to past resources, as well as any bias among the reviewers, does not play a role in the final selection process. This shift in approaches to funding on its own will not change the current power structure to be more inclusive, nor will any of the singular behaviors detailed in this chapter. Collectively, however, they usher in a new view on how to conduct science, and if taken as a full system could potentially lead to major changes.

\section{Representation of Samples}

The lack of diversity in research samples has been robustly documented for many years (Graham, 1992; Hartmann et al., 2013; Syed et al., 2018; see also Hamby, this volume), so we will not go into detail about this problem here other than to note that participants in psychological studies tend to be both White and from the U.S. The "and" in the preceding sentence is important to attend to, as there are two broad categories of cultural/racial/ethnic inclusion that are often conflated but need to always be taken separately: racial/ethnic minority inclusion and international inclusion. Both forms of exclusion from psychological research have been long known and discussed, and unfortunately long ignored. The fact remains that the vast majority of research is conducted in the U.S. and Western Europe and, within those countries, the vast majority of research is conducted with White people.

These issues had been raised repeatedly for years, but gained prominent attention when Henrich et al. (2010) published their paper on psychology's reliance on WEIRD samples--individuals who come from Western, Educated, Industrial, Rich, and Democratic countries. As we have discussed multiple times before, it is notable--but rarely recognized--that the WEIRD paper has almost nothing to say about race and ethnicity. We all know there is nothing so practical as a good acronym (Syed, 2020a), but we should always be skeptical of convenient acronyms and how they can perpetuate inequalities. WEIRD is almost too good, which also means it is also almost certainly leaving out other important facets of diversity that should be giving equal attention. WWEIRD (Harden, 2018), with the extra W for White, does not have the same cachet, nor does MWEIRD ("majority") or WEIRDER ("ethnic and racial" majorities). As usual, there is simply no space for ethnic/racial minorities.
Some readers may take a "who cares" attitude towards this issue of terminology, but it has real implications. WEIRD is complicit in the conflation of minority and international diversity and representation. Researchers will casually discuss the "WEIRD people problem" as though it is the same as diversity more generally (e.g., Muthukrishna \& Henrich, 2019), but WEIRD is narrowly focused on national diversity.

All of this is to say that the issues of representation of samples goes well beyond the "WEIRD people problem," and from a cultural psychology perspective we believe we would all be better off if we moved beyond WEIRD and named the problem for what it actually is. That is, it is the problem of a lack of diversity and inclusion within a power-based structure.

\section{How Greater Synergy between Open Science and Cultural Psychology Can Contribute to Represen- tation of Samples}

Aligning open science and cultural psychology, however, has the potential to address the problem in meaningful ways. We highlight two aspects here, collaboration and data sharing.

Collaboration. The fact that quantitative studies in psychology do not have sufficient statistical power has been long known and-like the lack of diversity of samples - long ignored (Cohen, 1962). Only recently, as part of the open science movement, has statistical power begun to receive sufficient attention. In this context, those who conduct research with ethnic minorities will quickly argue that recruiting a large enough sample to achieve statistical power is a burden (Gaither, 2019; Lewis, 2017).

Whereas it is certainly true that recruiting a larger number of ethnic minority participants will be, on average, more difficult than recruiting White participants, we need to attend to the existing research that is successful in this work and also work to think of new solutions. Both Gaither (2019) and Lewis (2017) discuss these difficulties in the context of using university participant pool samples, where there are sometimes too few ethnic minorities, and using online recruitment systems, where recruiting ethnic minorities can be expensive. As social psychologists it is not surprising that they would highlight these two recruitment methods, but these are not the only options.

Drawing from the democratic values that underlie the open science movement, collaboration has been a core aspect of the new scientific process (Forscher et al., 2020). Whereas it has long been the case that work was valued for being sole-authored, there is growing 
interest in shunning that view in favor of valuing work that is collaborative. Indeed, such a view is the idea of recognizing contributorship rather than authorship, which recognizes the many ways in which researchers can contribute to a final published report (Holcombe, 2019).

There are several new collaborative systems up and running in psychology in the wake of the open science movement. Most prominently is the Psychological Science Accelerator (Moshontz et al., 2018), "a globally distributed network of psychological science laboratories (currently over 500), representing over 70 countries on all six populated continents, that coordinates data collection for democratically selected studies " (https://psysciacc.org/). With this scale of infrastructure the researchers are able to address major questions related to diversity-oriented research, such as variation in evaluation of faces around the world (Jones et al., 2020) and the robustness of stereotype threat (Forscher et al., 2019).

Not all collaborations need be at the scale of the Psychological Science Accelerator, however, especially when researchers have interest in pursuing specific research questions that may not be shared with such a large number of labs. In the mid-2000s, a group of U.S. researchers with interests in identity, culture, and ethnicity developed the Multisite University Study of Identity and Culture (MUSIC; Weisskirch et al., 2013). The MUSIC collaborative began with a small group of researchers with shared interests, and over a couple of years expanded to include data from over 10,000 students at 30 institutions. MUSIC was a full-on democratic collaborative, with no principal investigator. Members interested in using the data for a paper contacted all other members with the plan, giving all the opportunity to join on provided they contribute based on pre-determined criteria (see Weisskirch et al., 2013 for a copy of the Memorandum of Understanding). The collaborative led to dozens of high-powered publications, presentations, and theses that otherwise would not have been. Moreover, the collaborative had very large representations of ethnic/racial minority researchers, early-career researchers, and those coming from smaller, under-resourced institutions. Remarkably, all of this was done with essentially no funding at all (see the first and second iterations of the Emerging Adults Measured at Multiple Institutions Project for a similar approach (Grahe et al., 2018; Reifman \& Grahe, 2016). To be sure, although it was inclusive, the collaborative relied on traditional approaches to authorship rather than a contributorship model, and thus did not fully address the incentive issues discussed previously. Nevertheless, the MUSIC collaborative is a nice example of cultural psychology and open science working in synergy to improve research practice: interest in cultural psychology paired with restricted access to participant populations led to broad and inclusive collaborations, which then led to stronger research being produced.

The issue of under-resourced institutions deserves special attention (Grahe et al., 2020). Most research in the U.S. comes out of large public land-grant universities or research-intensive private institutions. Such institutions are also not known for their diverse student bodies. Thus, if researchers at those institutions are relying only on their own participant pools and student bodies, then they may indeed have difficulty recruiting a suitable sample. But those institutions are not the be-all, end-all of higher education. Within reasonable proximity there are often regional state universities and definitely community colleges, both of which will tend to have much more diverse student populations. Engaging in genuine collaborations and partnerships (Cooper et al., 2005) between faculty at multiple institutions can diversify samples, provide access to research opportunities for students who may not otherwise have it, and creates a more inclusive and participatory field. Such an approach is consistent with the democractic ideals of the open science movement, and exemplifies how cultural psychology and open science can mutually benefit each other.

Data Sharing. A big part of the open science movement is the push for openly available data. Making data open has many scientific benefits; it allows others to evaluate the rigor of claims made in published work through reproducing the analysis that was conducted to ensure there are no errors or to specify alternative models that assess the robustness of the findings. Moreover, open data, has a generative function, allowing researchers to test different questions from those reported in published work. For example, Onyeador et al. (2020) published a three-wave longitudinal study of how contact with Black people was associated with changes in implicit and explicit bias in a large sample of physicians. The authors made their data openly available, which then allowed Schimmack (2019) to fit models that addressed the stability of implicit and explicit bias over time, a question that was not directly addressed in the original study or elsewhere in the literature. A cultural psychological project that engaged in open science practices allowed new questions about cultural psychology to be examined. 
In the context of psychological research, in which data sharing has not been the norm, the idea of making data openly available makes many researchers uneasy. This may be especially true for researchers working with ethnic minority populations, who may have a heightened sense of data privacy given the history of scientific racism. Indeed, many researchers underestimate the ability of re-identification of participants through their data, and this is an even higher risk for ethnic minority participants given their smaller size in the population. Moreover, researchers may collect large data sets that can be used for multiple purposes, and therefore do not want to share their data with others who may "scoop" them.

These are legitimate concerns that should be carefully considered, but they should not be used as de facto rationale for not sharing data. It is important to understand that "open data" is not a binary--open or not open--but rather can be a matter of degree. On one extreme end is fully open data, in which a complete data set is available online for public download without restriction. On the other end is fully closed data, where the data set is retained only by the original researchers and not made available to others. Interestingly, research suggests that agreements to make data available upon request are very rarely honored (Miyakawa, 2020; Statham et al., 2020; Wicherts et al., 2006) and are thus more or less equivalent to fully closed data.

But, again, there are middle positions between these two extremes that can address some concerns researchers might have about sharing data. First, researchers might publish an article that is based on a subset of the full data set, with plans to continue to work with variables not included in the published study. In this case, researchers may be reluctant to share their full data for fear of being scooped. One simple solution to this concern is to share only the data that were included in the analyses reported in the published article. Doing so would allow interested parties to reproduce the analyses and/or fit alternative models based on different assumptions.

A second concern about making data open is the risk of re-identification (Lewis, 2019). This may be a particularly salient concern for researchers working with ethnic minorities or other marginalized populations that, due to their minority status, may be at a heightened risk for re-identification. There are a variety of strategies for reducing the possibility of re-identification (Meyer, 2018). One straight-forward approach is to create two versions of the master data set, one with participants' demographics and one without.
The data set without the demographics could be made openly available, along with a codebook that indicates which demographics are included in the data set, summary data for the demographics, and a note that interested parties can contact the researchers for access to the demographics if needed. If researchers properly curate their data sets in the first place (Arslan, 2019), prior to posting the openly available version, then providing the full version upon request will not pose an administrative burden. An alternative emerging approach is to create a synthetic data set that mirrors the target data set so that researchers can reproduce the analyses or fit alternative models, but the data do not actually belong to any individuals and thus there is no risk of re-identification (Quintana, 2019).

Another approach to handling the re-identification concern is to make the data fully open and available upon request, but actually having a method for how authors can access the data. For example, researchers could create a data access and use agreement that interested parties had to complete prior to receiving the data. This agreement could have clear information about data privacy and reuse. Indeed, depositing the data at a trusted repository such as the Inter-university Consortium for Political and Social Research (ICPSR; www.icpsr.umich.edu/) would allow for them to set this kind of thing up for you.

The main take home point from the preceding is that making data openly available is more complex than it may seem, for better or for worse. We highly recommend reading Meyer (2018) for an excellent treatment of the complexities of data sharing and details on some of the repositories that are available.

\section{Representation of Perspectives}

The final form of representation pertains to the perspectives that researchers bring to their work, specifically theoretical and conceptual perspectives regarding diversity and groups that they may or may not be aware that they are drawing from.

Diversifying samples is often seen as an issue of external validity and generalization, that if our samples more adequately reflect society as a whole then we can be more confident in our claims about the generalizability of the findings. In this way, diversifying samples is largely a methodological change that improves the confidence of our conclusions. But this methodological change has serious substantive implications that are not always fully appreciated. 
Diversifying the samples almost always also means needing to test for variations in the core analyses along the major dimensions of diversity (e.g., testing for racial/ethnic group differences). Importantly, this need not always be the case, but it tends to be in practice because a) researchers work from the starting assumption that there are differences and thus they should be tested or b) reviewers work from the same starting assumption and require the authors to conduct such tests. So, generally, having a diverse sample means conducting tests for variations along that dimension of diversity.

But what if there is no theory or prior expectations for group differences? What if the only rationale is procedural ("have diversity must test") and not substantive? It turns out that we actually have few quality theories or generally substantive reasons to expect group variations in much of what we study. So then what we are left with is conducting exploratory analyses, which is fine, but what if we find differences? How do we make sense of them? Most researchers who test for racial/ethnic group differences, for example, are ill-equipped to do comparative racial/ethnic research because they did not think through from the beginning why such differences might be observed and ensure that they were measuring those explanatory constructs. Rather, researchers observe differences but have no idea why they found them, leading to post-hoc explanations that are of little benefit and are potentially harmful (see Helms et al., 2005, for a classic and still very relevant treatment of this issue).

If researchers find racial/ethnic group differences but are not sure why, in the next study they would hopefully be motivated to include and test for potential explanatory mechanisms. But to do so they would have to first delve deeper into ethnic minority psychology to examine culturally relevant mechanisms (e.g., identity, discrimination, socialization). At that point the researchers are then basically doing ethnic minority psychology, or more generally cultural psychology. And here they were just trying to get a more representative sample for their research!

This is why we must fully think through the recommendations to diversify our samples. It cannot stop there. Doing so actually requires us to diversify our thinking about psychological science. There is a substantive requirement attached to the methodological one. In fact, the larger issue is that thinking this through exposes the limited potential of the mainstream, universalist psychology. It highlights how we all are —or should be - cultural psychologists who take seriously the potential for systematic human variation in psychological phenomena.

First, there is a need to recognize the different cultural models that underlie any cultural-comparative inquiry. In a classic yet underappreciated chapter, Cauce et al. (1998) discuss three predominant models of cultural comparison: cultural deviance, cultural equivalence, and cultural variance. In the cultural deviance model, one group (typically the majority group) is set as the "standard" or "default" group to which others other are compared. Any deviation from the default group is considered to be a deficiency of the group, which is why such comparative approaches are often called "deficit models." In contrast, the cultural equivalence model rejects the assumption that one group is the default or normative standard, and assumes that any observed group differences are located within social-structural conditions. Accordingly, if not for those conditions then the groups could be equal. A common methodological approach within this model is to statistically control for social class when making racial/ethnic group comparisons. This is done to ostensibly make the groups equivalent, to remove the confound of social class, but it also assumes that social class has the same meaning across racial/ethnic groups, an assumption that has been demonstrated false (e.g., Hardaway \& McLoyd, 2009). Finally, the cultural variations models is free of assumptions about normative standards or the nature of equality. Rather, the assumption is that there are cultural variations among groups that may be associated with both differences and similarities among those groups. This approach seeks to take seriously the potential role of cultural and social-structural factors, but leaves room for such factors to have little or no impact on psychological phenomena. Awareness of the three and models, and which one is underlying researchers' thinking about their theory, design, and analysis is essential for conducting quality comparative work, and not taking the baseless view that all comparative work is problematic.

Identifying the cultural model is the first step to working through the next problem: identifying the mechanism that would account for any observed group differences. Most, but certainly not all, groupcomparison research hypothesizes the presence rather than absence of group differences (but see Chuard et al., 2019). At the same time, most, but certainly not all, of such research does not measure and test the putative mechanism that accounts for those differences. In classic examples, Helms at el. (2005) noted the lack of mechanisms included in Black-White comparisons 
of IQ and Matsumoto (1999) on the lack of mechanisms included in U.S.-Japan comparisons of independent and interdependent self-construals - critiques that are still highly relevant today.

As nicely summarized by $\mathrm{Ng}$ et al. (2019), conducting explanatory models for mean comparisons is accomplished through tests of mediation, whereas explanatory models of group differences between patterns of associations is accomplished through mediated moderation. Although the authors provide simulation-based power analyses that suggest Ns of 200300 being sufficient, their analysis was based on rather large effect sizes, and more reasonable power analyses suggest the need for a sample size of at least 400 under the most favorable conditions and up to 1100-1600 under other conditions (Giner-Sorolla, 2018; Simonsohn, 2014).

\section{How Greater Synergy between Open Science and Cultural Psychology Can Contribute to Represen- tation of Perspectives}

One of the aspects of open science that can potentially be most helpful for working through these theoretical issues is preregistration. Preregistration refers to the process of formally specifying your study design and analysis and submitting it to a trusted, timestamped repository before you collect the data and/or conduct the analysis (Nosek et al., 2018). The primary purpose is to draw a clearer line between confirmatory research--what you planned to do prior to seeing the data--and exploratory research--what you decided to do after seeing the data. Preregistration is meant to deter $\mathrm{p}$-hacking/questionable research practices/researcher degrees of freedom, which all correspond to the same patterns of conscious and unconscious modification of study design and analysis in order to achieve a p-value below the conventional .05 threshold. These practices lead to inflated Type 1 errors and lessened likelihood that the effect would replicate in an independent replication. Preregistration is also meant to deter HARKing--Hypothesizing after the results are known (Kerr, 1998)--because all hypotheses would be formally stated prior to the data analysis phase.

Preregistration is sometimes portrayed as a procedural step, or to ensure the veracity of the reported inferential statistics, but its theoretical value is often under-appreciated. The greatest value of developing preregistration plans, in our experience, is that it makes you stop and think. You have to think through, why am I doing this? Why am I making these choices, and what am I missing? Thinking through your predictions will require you to confront what to do about group differences - and thus, cultural psychology can help with creating stronger preregistration plans. First, is the decision about whether or not you will test for group differences, and if so, is it purely exploratory or do you have specific hypotheses about the reasons those differences might be observed? If the latter, then you can be sure that you include appropriate measures so that you can conduct the test. Contrast this approach to the more standard approach, where researchers realize they have a diverse sample and decide to test for differences, having no substantive rationale for doing so. The results of such tests are nearly impossible to interpret.

Having a preregistration plan indicating that you will not plan to test for group differences can also protect against editors and reviewers who insist on such tests (and they most certainly do). To be clear, you can always conduct analyses that were not part of the preregistration plan and include them clearly labeled as exploratory/post-hoc, but the plan help by having clearly specified central questions and how they were to be examined.

An extended version of preregistration are registered reports (Chambers \& Tzavella, 2020). With registered reports, authors submit the introduction, method, and planned analyses section for review, before collecting and/or analyzing any data. That is what is reviewed and initially accepted for publication. Then the authors conduct the study and/or analyze the data, and so long as they did what they said they would, and did so competently, then the article will be published regardless of the results. The idea behind registered reports is that selections for publication should be based on sound conceptualization, design, and analysis, not on the nature of the findings, which is the current mainstream approach that has led to massive publication bias. With registered reports, the idea and plan of execution is what gets rewarded. In our view this publishing format should be a boon for researchers working on diversity-related topics. As the registered report editors of Developmental Science recently discussed (Ansari \& Gervain, 2020), the format benefits those working with resource constraints, as it allows them to conduct a single, well-designed study rather than having to conduct numerous studies until they can produce results that are deemed suitable for publication. As the authors say, "This should therefore allow smaller laboratories, researchers from underprivileged areas and less senior scholars with fewer 
resources to have a better opportunity to publish." (see also Grahe et al., 2020).

\section{Conclusion: Integrating the Three Dimensions of Representation}

The purpose of this chapter was to bring open science and cultural psychology closer together, highlighting how they can benefit one another. In doing so, we focused on three types of representation: researchers, samples, and perspectives. These three dimensions of representation are clearly related but also not entirely dependent on each other; one can increase one of the three without a concomitant increase in the other. For example, as a field we could diversify the researchers, but those researchers will not necessarily conduct work committed to diversifying samples, or could adopt a cultural deviance perspective that maintains the current structure. Similarly, diversifying samples does not, technically, rely on diversifying researchers at all (although in practice it seems to; see Roberts et al. 2020).

That said, all three forms of representation can and often will have reciprocal relations with one another. For example, ethnic minority researchers in psychology will often - although of course not always - seek to conduct research with ethnic minority populations. The more the research in the field engages with diversity in its samples, potentially more likely that ethnic minorities see themselves as belonging in the field, enhancing recruitment and retention. Thus, a reciprocal relation between diversity of researchers and diversity of samples. Relying on more diverse samples requires researchers to think more deeply about what they are doing with those samples. Thinking more deeply about diversity in our work may lead to diversifying our samples to conduct research that is consistent with that thinking. Thus a reciprocal relation between diversity of samples and diversity of perspectives. Taking up different cultural perspectives may allow us to see the field from different angles, including from a structural one, and therefore could lead to field-wide action that increases the diversity of researchers. Increasing the number of ethnic minority researchers will likely diversify the cultural perspectives that underlie work in the field. Thus, a reciprocal relation between diversity of perspectives and diversity of researchers.

So yes, the dimensions clearly have the potential to be related, and can be conceptualized as part of a broader system. However, in terms of engaging with initiatives that would increase diversity or seek to address diversity issues in open science, those initiatives need to be targeted to each dimension specifically. If someone is talking about one dimension, and in response someone brings up another dimension, without full acknowledgement that they are talking about different issues, then that is an unproductive conversation. The dimensions must be disaggregated and discussed separately. Indeed, as highlighted throughout this chapter, each of the dimensions of representation has different implications, problems, and solutions with respect to open science.

We are not arguing that these three types are the only way to think about diversity, or that there are not entirely different ways to think about the complexities of this issue. Rather, separating the three dimensions is at least a useful starting point to drawing attention to some issues that we should be focusing on. Focusing on no singular issue outlined in this chapter will change the view on how to conduct science; however, collectively if taken as a full system could potentially lead to major changes. Accordingly, we see this chapter as a starting point for researchers in cultural psychology and open science to think about these issues, and an invitation to take these ideas further or in different directions, all in the service of creating a more inclusive and robust science.

\section{References}

Albornoz, D., \& Chan, L. (2018). Power and Inequality in Open Science Discourses 1 Poder e desigualdade nos discursos da Ciência Aberta. 4(1), 10.

Allen, C., \& Mehler, D. M. A. (2019). Open science challenges, benefits and tips in early career and beyond. PLOS Biology, 17(5), e3000246. https://doi.org/10.1371/journal.pbio.3000246

Ansari, D., \& Gervain, J. (2020). Two Years on: Registered Reports in Developmental Science. https://www.wiley.com/network/researchers/researcher-blogs/two-years-in-how-registered-reportsare-impacting-developmental-psychology-research-in-developmental-science

Arslan, R. C. (2019). How to Automatically Document Data With the codebook Package to Facilitate Data Reuse. Advances in Methods and Practices in Psychological Science, 2(2), 169-187. https://doi.org/10.1177/2515245919838783

Bahlai, C., Bartlett, L., Burgio, K., Fournier, A., Keiser, C., Poisot, T., \& Whitney, K. (2019). Open Science Isn't Always Open to All Scientists. American Scientist, 107(2), 78. https://doi.org/10.1511/2019.107.2.78

Baumeister, R. F. (2016). Charting the future of social psychology on stormy seas: Winners, losers, and recommendations. Journal of Experimental Social Psychology, 66, 153-158. https://doi.org/10.1016/j.jesp.2016.02.003

Cauce, A. M., Cornonado, N., \& Watson, J. (1998). Conceptual, methodological, and statistical issues in culturally competent research. In M. Hernandez \& M. R. Isaac (Eds.), Systems of care for children's mental health. Promoting cultural competence in 
children's mental health services (pp. 305-329). Paul H Brookes Publishing Co.

Chambers, C. D., \& Tzavella, L. (2020). Registered Reports: Past, Present and Future [Preprint]. MetaArXiv. https://doi.org/10.31222/osf.io/43298

Chan, L., Okune, A., Hillyer, B., Albornoz, D., \& Posada, A. (2019). Contextualizing Openness: Situating Open Science. 354.

Chuard, P. J. C., Vrtílek, M., Head, M. L., \& Jennions, M. D. (2019). Evidence that nonsignificant results are sometimes preferred: Reverse P-hacking or selective reporting? PLOS Biology, 17(1), e3000127. https://doi.org/10.1371/journal.pbio.3000127

Cohen, J. (1962). The statistical power of abnormal-social psychological research: A review. The Journal of Abnormal and Social Psychology, 65(3), 145-153. https://doi.org/10.1037/h0045186

Cole, E. R. (2009). Intersectionality and research in psychology. American Psychologist, 64(3), 170-180. https://doi.org/10.1037/a0014564

Cooper, C. R., Chavira, G., \& Mena, D. D. (2005). From Pipelines to Partnerships: A Synthesis of Research On How Diverse Families, Schools, and Communities Support Children's Pathways Through School. Journal of Education for Students Placed at Risk (JESPAR), 10(4), 407-430. https://doi.org/10.1207/s15327671espr1004_4

Corker, K. S. (2018). Open Science is a Behavior. https://cos.io/blog/open-science-is-a-behavior/

Crenshaw, K. (1993). Mapping the Margins: Intersectionality, Identity Politics, and Violence against Women of Color. Stanford Law Review, 43(6), 1241-1300.

Crüwell, S., van Doorn, J., Etz, A., Makel, M. C., Moshontz, H., Niebaum, J. C., Orben, A., Parsons, S., \& Schulte-Mecklenbeck, M. (2019). Seven Easy Steps to Open Science. Zeitschrift Für Psychologie, 227(4), 237-248. https://doi.org/10.1027/21512604/a000387

Delucio, K., \& Villicana, A. J. Intersectionality as an analytic sensibility in cultural research. In K. C. McLean (Ed). Cultural Methods in Psychology: Describing and Transforming Cultures. New York: Oxford University Press.

Fish, J., \& Syed, M. (2018). Native Americans in Higher Education: An Ecological Systems Perspective. Journal of College Student Development, 59(4), 17.

Fiske, A. P. P. S. T. (2016). A Call to Change Science's Culture of Shaming. APS Observer, 29(9). https://www.psychologicalscience.org/observer/a-call-to-change-sciences-culture-ofshaming

Forscher, P. S., Taylor, V. J., Cavagnaro, D., Lewis Jr, N., Moshontz, H., Batres, C., Mark, A. Y., Appleby, S., Bennett-Day, B., \& Buchanan, E. M. (2019). A multi-site examination of stereotype threat in black college students across varying operationalizations.

Forscher, P. S., Wagenmakers, E. J., DeBruine, L., Coles, N., Silan, M. A., \& IJzerman, H. (2020). A manifesto for team science. PsyArXiv. https://psyarxiv.com/2mdxh

Gaither, S. (2019, August 7). Diversifying Psychological Science. Psychology Today. https://www.psychologytoday.com/blog/different-identities-different-perspectives/201908/diversifying-psychological-science

Gelman, A. (2016). What has happened down here is the winds have changed. https://statmodeling.stat.columbia.edu/

Giner-Sorolla, R. (2018, January 24). Powering Your Interaction. Approaching Significance. https://approachingblog.wordpress.com/2018/01/24/powering-your-interaction-2/

Goodnow, J. J. (2011). Merging cultural and psychological accounts of family contexts. In L. A. Jensen (Ed.), Bridging cultural and developmental approaches to psychology: New syntheses in theory, research, and policy. Oxford University Press.

Graham, S. (19921001). "Most of the subjects were White and middle class": Trends in published research on African Americans in selected APA journals, 1970-1989. American Psychologist, 47(5), 629. https://doi.org/10.1037/0003-066X.47.5.629

Grahe, J. E., Chalk, H. M., Alvarez, L. D. C., Faas, C. S., Hermann, A. D., \& McFall, J. P. (2018). Emerging Adulthood Measured at Multiple Institutions 2: The Data. Journal of Open Psychology Data, 6(1), 4. https://doi.org/10.5334/jopd.38

Grahe, J. E., Cuccolo, K., Leighton, D. C., \& Cramblet Alvarez, L. D. (2020). Open Science Promotes Diverse, Just, and Sustainable Research and Educational Outcomes. Psychology Learning \& Teaching, 19(1), 5-20. https://doi.org/10.1177/1475725719869164

Hall, G. C. N., Martinez, C. R., Tuan, M., McMahon, T. R., \& Chain, J. (2011). Toward ethnocultural diversification of higher education. Cultural Diversity and Ethnic Minority Psychology, 17(3), 243-251. https://doi.org/10.1037/a0024036

Hamby, S. Strengths-based Approaches to Conducting Research with Low Income and Other Marginalized Populations. In K. C. McLean (Ed). Cultural Methods in Psychology: Describing and Transforming Cultures. New York: Oxford University Press.

Hardaway, C. R., \& McLoyd, V. C. (2009). Escaping Poverty and Securing Middle Class Status: How Race and Socioeconomic Status Shape Mobility Prospects for African Americans During the Transition to Adulthood. Journal of Youth and Adolescence, 38(2), 242-256. https://doi.org/10.1007/s10964-008-9354-z

Harden, P. (2018, October 27). Heredity is only half the story. The Spectator. https://www.spectator.co.uk/2018/10/heredity-isonly-half-the-story/

Hartmann, W. E., Kim, E. S., Kim, J. H. J., Nguyen, T. U., Wendt, D. C., Nagata, D. K., \& Gone, J. P. (2013). In Search of Cultural Diversity, Revisited: Recent Publication Trends in Cross-Cultural and Ethnic Minority Psychology. Review of General Psychology, 17(3), 243-254. https://doi.org/10.1037/a0032260

Helms, J. E., Jernigan, M., \& Mascher, J. (2005). The meaning of race in psychology and how to change it: A methodological perspective. American Psychologist, 60(1), 27-36. https://doi.org/10.1037/0003-066X.60.1.27

Henrich, J., Heine, S. J., \& Norenzayan, A. (2010). The weirdest people in the world? Behavioral and Brain Sciences, 33(2-3), 6183. https://doi.org/10.1017/S0140525X0999152X

Holcombe, A. O. (2019). Contributorship, Not Authorship: Use CRediT to Indicate Who Did What. Publications, 7(3), 48. https://doi.org/10.3390/publications7030048

Hunt, M. O., Jackson, P. B., Powell, B., \& Steelman, L. C. (2000). Color-Blind: The Treatment of Race and Ethnicity in Social Psychology. Social Psychology Quarterly, 63(4), 352-364. JSTOR. https://doi.org/10.2307/2695845

Inbar, Y. (2016). Association between contextual dependence and replicability in psychology may be spurious. Proceedings of the National Academy of Sciences, 113(34), E4933-E4934.

Jackson, S. J. (2019). Twitter made us better: A decade of mistrust. New York Times. Retrieved from: https:/www.nytimes.com/interactive/2019/12/27/opinion/sunday/twitter-social-media.html

Jackson, S. J., Bailey, M., \& Welles, B. F. (2020). \#HashtagActivism: Networks of race and gender justice. MIT Press.

Juang, L. P., Syed, M., Cookston, J. T., Wang, Y., \& Kim, S. Y. (2012). Acculturation-based and everyday family conflict in Chinese American families. New Directions for Child and Adolescent Development, 2012(135), 13-34. https://doi.org/10.1002/cd.20002 
Kathawalla, U. K., Silverstein, P., \& Syed, M. (2019). Easing into open science: A tutorial for graduate students. PsyArXiv. https://psyarxiv.com/vzjdp/

Kerr, N. L. (1998). HARKing: Hypothesizing After the Results are Known. Personality and Social Psychology Review, 2(3), 196217. https://doi.org/10.1207/s15327957pspr0203_4

Kuhn, T., S. (1962). The structure of scientific revolutions. University of Chicago Press.

Lewis, N. A. [NeilLewisJr]. (2017, June 4). Let's discuss this at @improvingpsych, please. Retrieved from https://twitter.com/NeilLewisJr/status/871425638388961282? $\mathrm{s}=20$

Lewis, N. A. (2017, August 11). Reflections on SIPS (guest post by Neil Lewis, Jr.). The Hardest Science. https://thehardestscience.com/2017/08/11/reflections-on-sips-guest-post-by-neillewis-jr/

Lewis, N. A. (2019). Open Communication Science: A Primer on Why and Some Recommendations for How. Communication Methods and Measures, 1-12. https://doi.org/10.1080/19312458.2019.1685660

Manago, A., Santer, N., Barsigian, L. L., \& Walsh, A. Social Media as Tools for Cultural Change in the Transition to Adulthood. In K. C. McLean (Ed). Cultural Methods in Psychology: Describing and Transforming Cultures. New York: Oxford University Press.

Markus, H. R., \& Kitayama, S. (1991). Culture and the self: Implications for cognition, emotion, and motivation. Psychological Review, 98(2), 224. https://doi.org/10.1037/0033-295X.98.2.224

Matsumoto, D. (1999). Culture and self: An empirical assessment of Markus and Kitayama's theory of independent and interdependent self-construals. Asian Journal of Social Psychology, 2(3), 289-310. https://doi.org/10.1111/1467-839X.00042

McBee, M. T., Makel, M. C., Peters, S. J., \& Matthews, M. S. (2018). A Call for Open Science in Giftedness Research. Gifted Child Quarterly, 62(4), 374-388. https://doi.org/10.1177/0016986218784178

Meyer, M. N. (2018). Practical tips for ethical data sharing. Advances in Methods and Practices in Psychological Science, 1(1), 131-144.

Milfont, T. L., \& Klein, R. A. (2018). Replication and Reproducibility in Cross-Cultural Psychology. Journal of Cross-Cultural Psychology, 49(5), $735-750$. https://doi.org/10.1177/0022022117744892

Miyakawa, T. (2020). No raw data, no science: Another possible source of the reproducibility crisis. Molecular Brain, 13(1), 24. https://doi.org/10.1186/s13041-020-0552-2

Moshontz, H., Campbell, L., Ebersole, C. R., IJzerman, H., Urry, H. L., Forscher, P. S., Grahe, J. E., McCarthy, R. J., Musser, E. D., \& Antfolk, J. (2018). The Psychological Science Accelerator: Advancing psychology through a distributed collaborative network. Advances in Methods and Practices in Psychological Science, 1(4), 501-515.

Muller Mirza, N., \& Dos Santos Mamed, M. (2019). Self-narration and agency as interactive achievements: A sociocultural and interactionist analysis of migrant women's stories in a language learning setting. Learning, Culture and Social Interaction, 21, 34-47. https://doi.org/10.1016/j.lcsi.2019.01.003

Muthukrishna, M., \& Henrich, J. (2019). A problem in theory. Nature Human Behaviour, 3(3), 221-229. https://doi.org/10.1038/s41562-018-0522-1

Nelson, L. D., Simmons, J., \& Simonsohn, U. (2018). Psychology's Renaissance. Annual Review of Psychology, 69(1), 511-534. https://doi.org/10.1146/annurev-psych-122216-011836

Ng, J. C. K., Chan, W., Kwan, J. L. Y., \& Chen, S. X. (2019). Unpacking Structure-Oriented Cultural Differences Through a Me- diated Moderation Model: A Tutorial With an Empirical Illustration. Journal of Cross-Cultural Psychology, 50(3), 358-380. https://doi.org/10.1177/0022022118821183

Nosek, B. A., Ebersole, C. R., DeHaven, A. C., \& Mellor, D. T. (2018). The preregistration revolution. Proceedings of the $\mathrm{Na}$ tional Academy of Sciences, 115(11), 2600-2606.

Nuijten, M. B. (2019). Practical tools and strategies for researchers to increase replicability. Developmental Medicine \& Child Neurology, 61(5), 535-539. https://doi.org/10.1111/dmcn.14054

Onyeador, I. N., Wittlin, N. M., Burke, S. E., Dovidio, J. F., Perry, S. P., Hardeman, R. R., Dyrbye, L. N., Herrin, J., Phelan, S. M., \& van Ryn, M. (2020). The Value of Interracial Contact for Reducing Anti-Black Bias Among Non-Black Physicians: A Cognitive Habits and Growth Evaluation (CHANGE) Study Report. Psychological Science, 31(1), 18-30. https://doi.org/10.1177/0956797619879139

Open Science Collaboration. (2015). Estimating the reproducibility of psychological science. Science, 349(6251), aac4716.

Paola Masuzzo. (2020, February 22). Open Science: The science of the future. https://doi.org/10.5281/zenodo.3679141

Purdie-Vaughns, V., \& Eibach, R. P. (2008). Intersectional Invisibility: The Distinctive Advantages and Disadvantages of Multiple Subordinate-Group Identities. Sex Roles, 59(5), 377-391. https://doi.org/10.1007/s11199-008-9424-4

Quintana, D. (2019). Synthetic datasets: A non-technical primer for the behavioural sciences to promote reproducibility and hypothesis-generation [Preprint]. PsyArXiv. https://doi.org/10.31234/osf.io/dmfb3

Reifman, A., \& Grahe, J. E. (2016). Introduction to the Special Issue of Emerging Adulthood. Emerging Adulthood, 4(3), 135-141. https://doi.org/10.1177/2167696815588022

Renkewitz, F., \& Heene, M. (2019). The Replication Crisis and Open Science in Psychology: Methodological Challenges and Developments. Zeitschrift Für Psychologie, 227(4), 233-236. https://doi.org/10.1027/2151-2604/a000389

Rogoff, B. (2003). The Cultural Nature of Human Development. Oxford University Press, USA.

Sakaluk, J. K. (2016). Promoting replicable sexual science: A methodological review and call for metascience. The Canadian Journal of Human Sexuality. https://doi.org/10.3138/cjhs.251-CO1

Schimmack, U. (2019, November 28). Racial Bias as a Trait. Replicability-Index. https://replicationindex.com/2019/11/28/racialbias-as-a-trait/

Segall, M. H., Lonner, W. J., \& Berry, J. W. (1998). Cross-Cultural Psychology as a Scholarly Discipline. American Psychologist, 10.

Shweder, R. A. (2000). The psychology of practice and the practice of the three psychologies. Asian Journal of Social Psychology, 3(3), 207-222. https://doi.org/10.1111/1467-839X.00065

Simons, D. J., Shoda, Y., \& Lindsay, D. S. (2017). Constraints on generality (COG): A proposed addition to all empirical papers. Perspectives on Psychological Science, 12(6), 1123-1128.

Simonsohn, U. (2014, March 12). [17] No-way Interactions. Data Colada. http://datacolada.org/17

Simonsohn, U. (2017, October 20). [63 ]"Many Labs" overestimated the importance of hidden moderators. Data Colada. http://datacolada.org/63.

Spellman, B. A. (2015). A Short (Personal) Future History of Revolution 2.0. Perspectives on Psychological Science, 10(6), 886899. https://doi.org/10.1177/1745691615609918

Spellman, B. A., Gilbert, E. A., \& Corker, K. S. (2018). Open Science. In Stevens' Handbook of Experimental Psychology and Cognitive Neuroscience (pp. 1-47). American Cancer Society. https://doi.org/10.1002/9781119170174.epen519 
Statham, E. E., White, S. A., Sonwane, B., \& Bierer, B. E. (2020). Primed to comply: Individual participant data sharing statements on ClinicalTrials.gov. PLOS ONE, 15(2), e0226143. https://doi.org/10.1371/journal.pone.0226143

Strack, F. (2017). From Data to Truth in Psychological Science. A Personal Perspective. Frontiers in Psychology, 8. https://doi.org/10.3389/fpsyg.2017.00702

Syed, M. (2017). Why Traditional Metrics May Not Adequately Represent Ethnic Minority Psychology. Perspectives on Psychological Science, 12(6), 1162-1165. https://doi.org/10.1177/1745691617709590

Syed, M. (2019). The Open Science Movement is For All of Us.

Syed, M. (2020a). Acronym absurdity constrains psychological science. PsyArXiv. https://psyarxiv.com/293wx

Syed, M. (2020b). The promise of the open science movement for research on identity. Identity.

Syed, M., \& Kathawalla, U.-K. (2018). Integrating Culture and Biology in Psychological Research: Conceptual Clarifications and Recommendations. In J. M. Causadias, E. H. Telzer, \& N. A. Gonzales (Eds.), The Handbook of Culture and Biology (pp. 3154). John Wiley \& Sons, Inc. https://doi.org/10.1002/9781119181361.ch2

Syed, M., Santos, C., Yoo, H. C., \& Juang, L. P. (2018). Invisibility of racial/ethnic minorities in developmental science: Implications for research and institutional practices. American Psychologist, 73(6), 812-826. https://doi.org/10.1037/amp0000294

Van Bavel, J. J., Mende-Siedlecki, P., Brady, W. J., \& Reinero, D. A. (2016). Contextual sensitivity in scientific reproducibility. Proceedings of the National Academy of Sciences, 113(23), 64546459.
Vicente-Saez, R., \& Martinez-Fuentes, C. (2018). Open Science now: A systematic literature review for an integrated definition. Journal of Business Research, 88, 428-436. https://doi.org/10.1016/j.jbusres.2017.12.043

Weisskirch, R. S., Zamboanga, B. L., Ravert, R. D., Whitbourne, S. K., Park, I. J. K., Lee, R. M., \& Schwartz, S. J. (2013). An introduction to the composition of the Multi-Site University Study of Identity and Culture (MUSIC): A collaborative approach to research and mentorship. Cultural Diversity and Ethnic Minority Psychology, 19(2), 123. https://doi.org/10.1037/a0030099

Whitaker. (2018, January 31). Open science: Sharing is caring, but is privacy theft? by David Mehler and Kevin Weiner | PLOS Neuroscience Community. https://blogs.plos.org/neuro/2018/01/31/open-science-sharing-iscaring-but-is-privacy-theft-by-david-mehler-and-kevin-weiner/, http://blogs.plos.org/neuro/?p=19527

Wicherts, J. M., Borsboom, D., Kats, J., \& Molenaar, D. (2006). The poor availability of psychological research data for reanalysis. American Psychologist, 61(7), 726-728. https://doi.org/10.1037/0003-066X.61.7.726

Wiernik, B. M. (2019, October 11). Preprint templates. 10.17605/OSF.IO/HSV6A

Wilson, S., Breen, A. V., DuPré, L. Mining for Culture or Researching for Justice? Unsettling Psychology through Indigenist Conversation. In K. C. McLean (Ed). Cultural Methods in Psychology: Describing and Transforming Cultures. New York: Oxford University Press.

Yarkoni, T. (2019, July 13). I hate open science. [Citation Needed]. https://www.talyarkoni.org/blog/2019/07/13/i-hate-open-science/ 